\title{
Unit Interval Time and Magnitude Monitoring Using Beta and Unit Gamma Distributions
}

\author{
Muhammad Farhan Akram, ${ }^{1}$ Sajid Ali $\mathbb{D},{ }^{1}$ Ismail Shah $\mathbb{D}^{1},{ }^{1}$ and Giulia Marcon ${ }^{2}$ \\ ${ }^{1}$ Department of Statistics, Quaid-i-Azam University, Islamabad 45320, Pakistan \\ ${ }^{2}$ Department of Engineering, University of Palermo, Viale delle Scienze, Palermo 90128, Italy \\ Correspondence should be addressed to Sajid Ali; sajidali@qau.edu.pk
}

Received 10 December 2021; Revised 25 December 2021; Accepted 15 January 2022; Published 21 February 2022

Academic Editor: Kenan Yildirim

Copyright (C) 2022 Muhammad Farhan Akram et al. This is an open access article distributed under the Creative Commons Attribution License, which permits unrestricted use, distribution, and reproduction in any medium, provided the original work is properly cited.

\begin{abstract}
Quick detection of an assignable cause is necessary for process accuracy with respect to the specifications. The aim of this study is to monitor the time and magnitude processes based on unit-interval data. To this end, maximum exponentially weighted moving average (Max-EWMA) control chart for simultaneous monitoring time and magnitude of an event is proposed. To be precise, beta and unit gamma distributions are considered to develop the Max-EWMA chart. The chart's performance is accessed using average run length (ARL), the standard deviation of run length (SDRL), and different quantiles of the run length distribution through extensive Monte Carlo simulations. Besides a comprehensive simulation study, the proposed charting methodology is applied to a real data set. The results show that the proposed chart is more efficient in detecting small to medium-sized shifts. The results also indicate that simultaneous shifts are detected more quickly as compared to the pure shift.
\end{abstract}

\section{Introduction}

Statistical quality control (SQC) is the science of using statistical techniques in maintaining and monitoring the quality level of products and services. There are two main components of SQC: acceptance sampling and statistical process control (SPC). Acceptance sampling is a technique of selecting a lot (of products, goods, and raw material) based on the result (or quality) of selected samples. On the other hand, SPC uses sampling along with other statistical methods to assess the quality of output of a manufacturing process. A graphical representation known as the control chart of the process helps to determine whether the process output achieves the acceptable quality level or not.

In SPC, monitoring of a process to check if it is incontrol (IC) or out-of-control (OOC) is done only after the stable process parameters, including mean, fraction nonconforming, or variance, are obtained. Control charts have long been and extensively used in the areas of manufacturing to monitor a process' stability, and recently, their applications have extended beyond the manufacturing processes.
For instance, control charts have been used to monitor the stability of stock price or the quality of a service. Furthermore, they are used in disease surveillance systems to assess whether a disease has reached to the pandemic level or not.

One of the techniques used in SPC to monitor a process is the Shewhart approach where the process is termed as IC or OOC based on the result of a current sample. This technique usually performs better when we have large size disturbances in a process. Since this technique completely ignores the past information (because of which this scheme is also called memoryless charting scheme), it cannot detect small shifts in the process. Contrary to Shewhart approach, the memory-type charting schemes (such as cumulative sum (CUSUM) and exponentially weighted moving average (EWMA)) consider not only the current but also the past information about the process. This feature enables these charts to detect small shifts in the process.

Many real-life settings involve data of rates or proportions, e.g., monitoring the ratio of ingredients of life-saving drugs, etc., and hence, there arises a need to develop the charting mechanism for such types of data. The data of rates 
and proportions may not be resulted from a Bernoulli process, and therefore, control charts other than the traditional attribute charts, such as $\mathrm{p}$ and $\mathrm{np}$ charts, are required. For instance, monitoring the rate of unemployment to ensure the better utilization of resources in public politics can be performed with these charts. While dealing with different processes, magnitude associated with an event occurrence plays an important role to define its quality. This study aims at presenting the simultaneous monitoring methodology of time and magnitude based on the distributions having support between 0 and 1 . To this end, the exponentially weighted moving average (EWMA) charting mechanism with a slight modification is used in order to develop the proposed chart. Different shift sizes are introduced, and the average speed, known as the average run length (ARL) to measure the average of the out-of-control signals, is used. The average run length is the most widely and frequently used criterion for evaluating the performance of a control chart. Our proposed chart is flexible and can be applied to many real-life scenarios.

For Bernoulli data, attribute control charts such as $p$ chart is applied to monitor the fraction and the number of nonconforming items, respectively [1]. An advantage of an attribute control chart over variable control chart is that it is economical and less time-consuming compared to the former because attribute data collection is much easier than variable data. Ho and Quinino [2] used an attribute control chart to monitor the variability of a process. There are a number of practical situations where one might be interested in the monitoring of proportions or rates in a product; however, the available data set may not follow the Bernoulli distribution [3]. The data of proportions and rates can be described as collection of observations where the variable of interest is confined to an interval of $(0,1)$. Such data can be found extensively in many fields of study and often analyzed using normal distribution because of simplicity associated with normal distribution, yet misleading conclusions [4].

Certain distributions are available in the literature which can be utilized to deal with proportion data. One of such distributions is known as the beta distribution. Sant'Anna and Ten Caten [5] considered this distribution along with Shewhart strategy to construct the beta control chart for monitoring fractional type variables of a manufacturing process. Later, Lima-Filho et al. [6] constructed an inflated beta control chart. Apart from beta distribution, the unit gamma (UG) can exhibit constant, unimodal, increasing, and decreasing shapes, whereas its hazard function has bathtub and decreasing shapes [7]. Grassia [8] gave a detailed overview of this distribution as well as of its variants. Ratnaparkhl and Mosimann [9] studied this distribution to derive some new distributions. The UG distribution is similar to the beta distribution in terms of some of the properties, and hence, some of its applications are the same as that of the beta distribution. This distribution can be used to estimate the density of virus or bacteria. Tadikamalla [10] showed that the UG distribution can be used as an alternative for Johnson SB and beta distributions. Recently, Mousa et al. [11] developed a regression model using this distribution.
Time-between events (TBE) charts have recently been the focus of many researchers as these charts are very effective in monitoring high quality processes such as high yield production lines with extremely small defect rates [12]. Several TBE control charts are available in literature [13]. These charts also overcome the drawbacks of traditional Shewhart charting schemes, including high false alarm rate, the assumption of normality, and negative lower limit. Ali [14] proposed the TBE control chart based on the renewal process assuming a continuous exponentiated family of distributions. The authors concluded that the proposed chart is more efficient and flexible when compared to existing TBE charts based on exponential distribution. For some recent contributions in this direction, we refer to the works of Xie et al. [15], Zhang et al. [16], Shafae et al. [17], Shah et al. [18], Talib et al. [19], and references cited therein.

Wu et al. [20] developed a rate chart for monitoring time and magnitude simultaneously by using the ratio of magnitude and time as the plotting statistic. Practically, during a certain time period, the effect of presence of an event, i.e., the damage or disturbance it can cause, is determined by its frequency or by its magnitude. Thus, there arises a need to monitor both of these characteristics (frequency and magnitude) simultaneously. Taking this into account, Wu et al. [21] adopted a Shewhart control charting technique for monitoring the frequency and magnitude of an event simultaneously. In earlier studies, simultaneous monitoring was done using two separate charting statistics. This technique was later found to be less effective when compared to the charts based on single statistic except for some exceptional situations. Most of the work on simultaneous monitoring is based on Shewhart mechanism [22, 23]. However, Shewhart charts are of memoryless nature, and the historical information of the process is ignored as the new points are plotted [24]. As a result, these charts cannot detect small shifts in process parameters satisfactorily. Consequently, memory-type control charts such as exponentially weighted moving average chart (EWMA) and cumulative sum (CUSUM) chart are required.

The EWMA chart is more sensitive as compared to the Shewhart chart when it comes to reflecting critical past information. In addition, unlike Shewhart and CUSUM schemes, the EWMA chart is not sensitive to nonnormality of data for smaller values of the smoothing parameter [25]. Most recently, Sanusi et al. [26] introduced the maximum EWMA (Max-EWMA) control chart to simultaneously monitor the frequency and magnitude of events where the frequency was assumed to follow the exponential and magnitude followed a gamma distribution.

The rest of the article is organized as follows. The probability density functions of the beta and unit gamma distributions with transformed parameters to have the same means are discussed in Section 2. Max-EWMA chart using the beta and unit gamma distributions is defined in Section 3. The charting procedure is also defined in the same section. Section 4 presents the in and out of control performance of the Max-EWMA chart. Real data examples are discussed in Section 5, while concluding remarks are given in Section 6. 


\section{Beta and Unit Gamma Distribution}

For the proposed Max-EWMA control chart, we consider the beta and unit gamma distribution in this section to provide in-depth analysis of the unit data monitoring problem.

2.1. Beta Distribution. A random variable $T$ follows a beta distribution with shape parameters $\delta>0$ and $\gamma>0$, if its probability density function (PDF) is given by

$$
f(t \mid \delta, \gamma)=\frac{t^{\delta-1}(1-t)^{\gamma-1}}{B(\delta, \gamma)}, \quad 0<t<1,
$$

where $B(\delta, \gamma)=(\Gamma(\delta) \Gamma(\gamma) / \Gamma(\delta+\gamma))$ is called the beta function and $\Gamma(\delta)=\int_{0}^{\infty} \omega^{\delta-1} e^{-\omega} \mathrm{d} t$. The mean and variance of $\mathrm{T}$ are

$$
\begin{aligned}
E(T) & =\mu=\frac{\delta}{\delta+\gamma}, \\
\operatorname{Var}(T) & =\frac{\delta \gamma}{(\delta+\gamma)^{2}(\delta+\gamma+1)},
\end{aligned}
$$

respectively. Suppose, we transform the parameters in equation (1) as

$$
\begin{aligned}
\delta & =\mu \phi, \\
\gamma & =(1-\mu) \phi .
\end{aligned}
$$

Now, using equation (3), the PDF of the beta distribution in equation (1) takes the form

$$
f(t \mid \delta, \gamma)=\frac{t^{\mu \phi-1}(1-t)^{(1-\mu) \phi-1}}{B(\mu \phi,(1-\mu) \phi)}, \quad 0<t<1 .
$$

Furthermore, the mean and the variance of $T$ with transformed parameters are $E(T)=\mu$ and $\operatorname{Var}(T)=(\mu(1-\mu) / \phi+1)$, respectively.

2.2. Unit Gamma Distribution. A random variable $\mathrm{X}$ follows the unit gamma distribution with shape parameter $\tau>0$ and rate parameter $\theta>0$, i.e., $X \sim u G(\tau, \theta)$, if it has the following PDF:

$$
f(x \mid \tau, \theta)=\frac{\theta^{\tau}}{\Gamma(\tau)} x^{\theta-1}\left[\log \left(\frac{1}{x}\right)\right]^{\tau-1}, \quad 0<x<1,
$$

The mean and variance associated with equation (5) are

$$
\begin{gathered}
E(X)=\mu=\left[\frac{\theta}{\theta+1}\right]^{\tau}, \\
\operatorname{Var}(X)=\left[\frac{\theta}{\theta+2}\right]^{\tau}-\left[\frac{\theta}{\theta+1}\right]^{2 \tau},
\end{gathered}
$$

respectively. Let us transform $\theta$ in the above PDF as $\theta=\left(\mu^{1 / \tau} /\left(1-\mu^{1 / \tau}\right)\right)$. Then, the PDF of X takes the form

$$
\begin{array}{r}
f(x \mid \tau, \theta)=\frac{\left[\left(\mu^{1 / \tau} / 1-\mu^{1 / \tau}\right)\right]^{\tau}}{\Gamma(\tau)} x^{\left(\mu^{1 / \tau} / 1-\mu^{1 / \tau}\right)-1}\left[\log \left(\frac{1}{x}\right)\right]^{\tau-1}, \\
0<x<1,
\end{array}
$$

where $\tau>0$ and $0<\mu<1$. Under this parameterization, we have $E(X)=\mu$ and $\operatorname{Var}(X)=\mu\left[\left(1 /\left(2-\mu^{1 / \tau}\right)^{\tau}\right)-\mu\right]$.

\section{Max-EWMA Control Chart Using Beta and Unit Gamma Distributions}

To jointly monitor unit interval time and magnitude, the Max-EWMA charting procedure is adopted. The plotting statistic of the Max-EWMA control chart consists of the maximum value of two independent EWMA statistics, both in absolute terms. For joint monitoring of the TBE (T) and magnitude $(\mathrm{X})$ of the event, the maximum of absolute values of TBE and magnitude is plotted. When an OOC signal is observed, the chart has the capability to identify whether the shift has occurred in T or X.

In particular, this study considers that the TBE follows the beta distribution, $T \sim B(\delta, \gamma)$, and magnitude of the event follows the unit gamma distribution, $X \sim u G(\tau, \theta)$. It is also assumed that the time between the occurrences of two successive disturbances and magnitude is independent. Furthermore, the process is assumed to start at time $t=0$, and the IC parameters are assumed to be $\left(\delta_{0}, \gamma_{0}\right)$ and $\left(\tau_{0}, \theta_{0}\right)$. Let the shifts in the parameters of $\mathrm{T}$ and $\mathrm{X}$, respectively, are represented as follows, $\delta=\Delta_{\delta} \delta_{0}, \gamma=\Delta_{\gamma} \gamma_{0}$, $\tau=\Delta_{\tau} \tau_{0}$, and $\theta=\Delta_{\theta} \theta_{0}$, respectively, where $\Delta_{\delta}, \Delta_{\gamma}, \Delta_{\tau}$, and $\Delta_{\theta}$ represent the shifts in the shape parameters of the beta distribution and shape and rate parameter of the unit gamma distribution, respectively. To this end, we have further considered two cases.

3.1. Case 1(a): Shifts in Shape Parameters of Beta and Unit Gamma Distribution. In the first case, shifts in the first shape parameter $\delta$ of the beta distribution and shape parameter $\tau$ of unit gamma distribution are considered. The process is termed as IC when both $\Delta_{\delta}$ and $\Delta_{\tau}$ are equal to one. The following two independent statistics are defined in the construction of the Max-EWMA chart

$$
\begin{aligned}
U_{i} & =\frac{X_{i}-\mu}{\sqrt{\mu\left[\left(1 /\left(2-\mu^{1 / \tau}\right)^{\tau}\right)-\mu\right]}}, \\
V_{i} & =\frac{T_{i}-\mu}{\sqrt{(\mu(1-\mu) / \phi+1)}} .
\end{aligned}
$$

The EWMA statistics, based on $U_{i}$ and $V_{i}$, are defined as

$$
\begin{array}{ll}
Y_{i}=\left(1-\lambda_{e}\right) Y_{i-1}+\lambda_{e} U_{i}, & 0<\lambda_{e} \leq 1, i=1,2, \ldots, \\
Z_{i}=\left(1-\lambda_{e}\right) Z_{i-1}+\lambda_{e} V_{i}, & 0<\lambda_{e} \leq 1, i=1,2, \ldots,
\end{array}
$$

where $\lambda_{e} \in(0,1]$ is one of the parameters of the EWMA control chart called the smoothing constant (the other parameter is known as the constant multiplier L). The starting 
values of two EWMA statistics are $Y_{0}=E\left(U_{i}\right)=0$ and $Z_{0}=E\left(V_{i}\right)=0$, respectively. Traditionally, a small value of $\lambda_{e}$ is considered to be suitable for detecting small shifts, whereas a large value is deemed appropriate to detect large shifts. Practically, $\lambda_{e} \in[0.05,0.25]$ is considered to detect small or medium-sized shifts in the process [27].

The monitoring statistic of the Max-EWMA control chart comprises the maximum of absolute of $Y_{i}$ and $Z_{i}$, i.e.,

$$
M_{i}=\max \left\{\left|Y_{i}\right|,\left|Z_{i}\right|\right\} .
$$

If there is shift in either $\mathrm{T}$ (shapel parameter of the beta distribution) or X (shape parameter of the unit gamma distribution) or in both parameters, the $M_{i}$ statistic becomes larger as compared to when there is no shift in either of the parameters. Furthermore, the proposed Max-EWMA chart only has the upper control limit (UCL) due to the fact that the plotting statistic is real and nonnegative number. The UCL is computed as

$$
\mathrm{UCL}=E(M)+L \sqrt{\operatorname{Var}(M)} .
$$

Here, $E(M)$ and $\operatorname{Var}(M)$ are the IC values of mean and variance of the plotting statistic, and $\mathrm{L}$ is the constant multiplier that controls the width of control limit. When $E(M)$ and $\operatorname{Var}(M)$ are unknown, one can replace them with sample mean and variance of $\mathrm{M}$, calculated from the IC historical data. The Max-EWMA chart can detect the upward shift (UW) shift as well as the downward (DW) shift in the $\mathrm{X}$ and/or $\mathrm{T}$ simultaneously.

3.2. Case 1(b): Shifts in the Shape Parameter of Beta Distribution and Rate Parameter of the Unit Gamma Distribution. In the second case, shifts in $\gamma$ parameter of the beta and $\theta$ parameter of the unit gamma distribution are considered. Similar to the first case, the process is IC when both $\Delta_{\gamma}$ and $\Delta_{\theta}$ are equal to one.

\subsection{Charting Procedure for the Max-EWMA Control Chart.} It is of utmost importance to identify the variables ( $\mathrm{T}$ and/or $\mathrm{X}$ ) being the cause of the OOC signal once it is triggered by the chart. As mentioned previously, follow-up approach, once an OOC state has been established, can be labelled easily using the Max-EWMA chart. The summary of the construction of the Max-EWMA control chart for simultaneous monitoring of frequency and magnitude is given as follows:

(1) Initialize $Y_{0}=Z_{0}=0$ and specify the desired $\mathrm{ARL}_{0}$ value. Also, select the suitable value of constant multiplier L and smoothing parameter $\lambda_{e}$ to achieve the desired $\mathrm{ARL}_{0}$. Different values of $\mathrm{L}$, for different combinations of $\tau, \mu$, and $\phi$ are given in Table 1 . The table values are obtained assuming $\mathrm{ARL}_{0} 370$ and 500. The table also includes the standard deviation of average run length along with different percentiles to access the skewness of the run length distribution.

(2) Calculate the value of UCL using equation (11). If $E(M)$ and $\operatorname{Var}(M)$ are not known, replace them with the corresponding sample values estimated from past data when the process does not contain any shift.

(3) Obtain the $i^{\text {th }}$ test observation, i.e., obtain $\left(X_{i}, T_{i}\right)$, where $X_{i}$ is $i^{\text {th }}$ observation of event's magnitude and $T_{i}$ is the corresponding TBE between the occurrences of event's $i^{t h}$ and $(i-1)^{\text {th }}$ observations.

(4) For $i^{\text {th }}$ test observation, compute the values of $M_{i}, U_{i}, V_{i}, Y_{i}$, and $Z_{i}$.

(5) Start monitoring the process by plotting the $M_{i}$ corresponding to the counter value $i$. The process is termed as the IC if $M_{i} \leq \mathrm{UCL}$, otherwise OOC. If the process is IC, monitoring continues; otherwise, proceed to the next step.

(6) Investigate the process to figure out if the process is really in an OOC state, if yes, take the necessary actions to eliminate the cause(s) behind an OOC signal. Resume the process of monitoring and move to step 3.

(7) To identify the OOC signal, label the points plotted above the UCL as per the symbols given in Table 2. If both $\left|Y_{i}\right|$ and $\left|Z_{i}\right|$ exceed UCL, use the label " $X T^{\text {" }}$ " to indicate shifts in both time and magnitude of the event. If only $\left|Y_{i}\right|$ exceeds UCL, use the label " $X$ " to indicate a shift in the magnitude only. Similarly, if $\left|Z_{i}\right|$ alone exceeds UCL, use the label " $T$ " to indicate a shift in TBE.

\section{Performance of the Proposed Chart}

The average run length (ARL) is the most common measure to access the performance of a control chart. It is defined as the average of run length, i.e., average number of subgroups (samples) plotted until an OOC signal is observed. Two types of ARL are defined in the literature: the in-control ARL $\left(\mathrm{ARL}_{0}\right)$ and the out-of-control ARL $\left(\mathrm{ARL}_{1}\right)$. Under ideal conditions, $\mathrm{ARL}_{0}$ should be as large as possible so that unnecessary pauses in the production line can be avoided. On the other hand, the smaller the value of $A R L_{1}$, the better the performance of the control chart. In fact, in comparative analysis, the chart with a smaller value of $\mathrm{ARL}_{1}$ is considered the best. Since the ARL distribution is skewed, we need to study its standard deviation (SDRL) as well as the percentiles (q10, q25, q50, q75, and q90) of the run length. Therefore, a detailed analysis of the ARL along with its standard deviation (SDRL) and different percentiles of run length distribution are given in Tables 3 and 4 and Table S1 (Supplementary Materials) corresponding to Case 1(a), and in Tables S2-S4 corresponding to Case 1(b). Even though our interest lies in the simultaneous monitoring of the magnitude and TBE, shifts in a single parameter of each distribution, keeping IC the corresponding parameter of other distribution, is also considered. For example, shift in shape 1 parameter of the beta distribution is considered in Table 3 keeping the shape parameter of the unit gamma distribution in-control. Furthermore, shift in shape parameter of unit gamma, keeping IC the shapel parameter of the beta distribution, is considered in Table 4. Finally, 


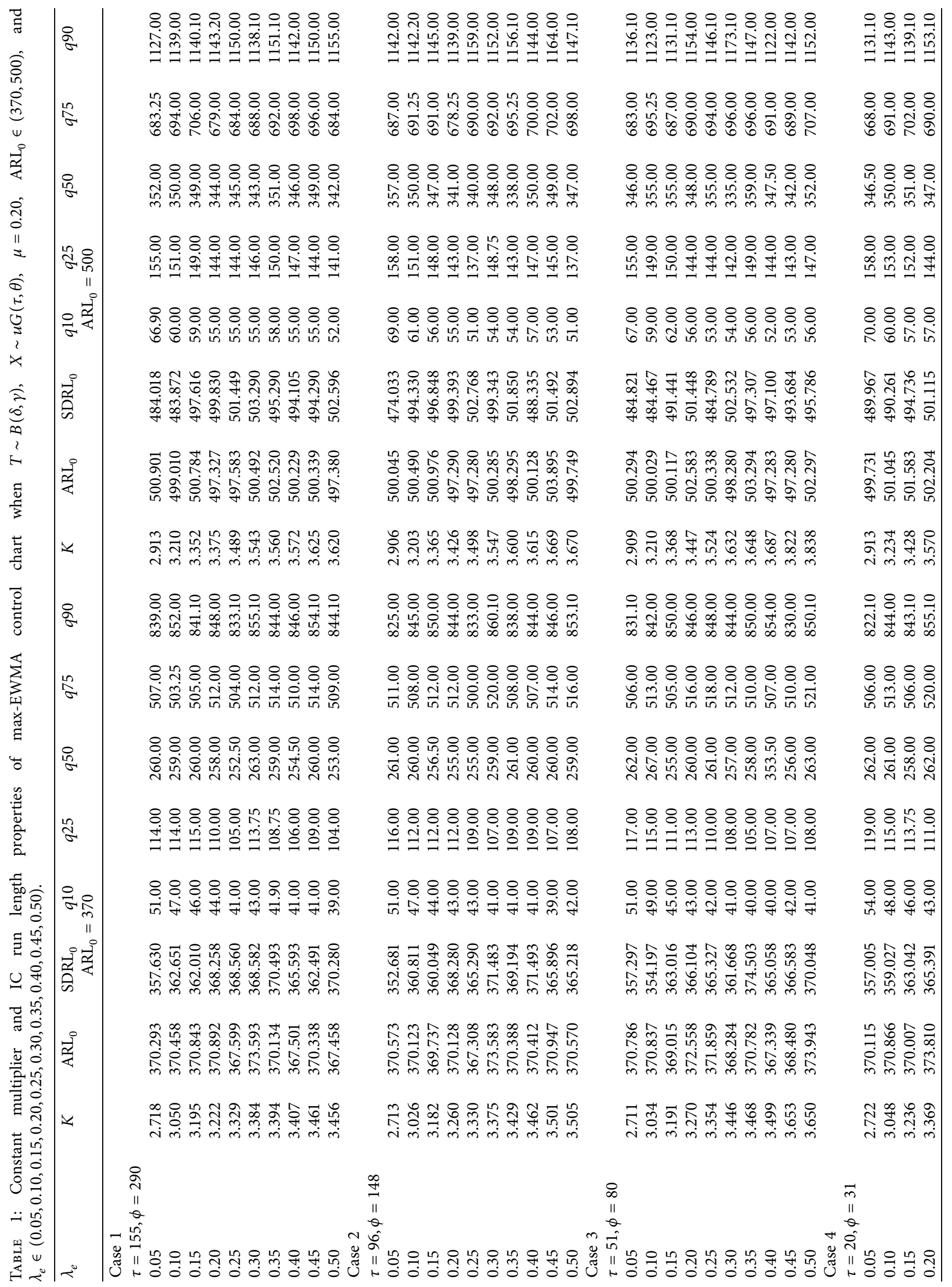




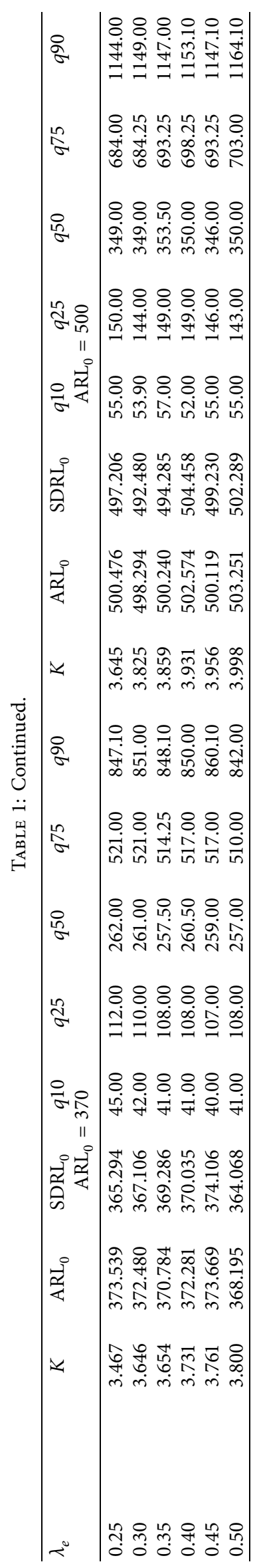


TABLE 2: Symbols representing the source of the out-of-control signal.

\begin{tabular}{lcc}
\hline Time & \multicolumn{3}{c}{ Magnitude } \\
& $|Y|<\mathrm{UCL}$ & $|Y|>\mathrm{UCL}$ \\
\hline$|Z|<\mathrm{UCL}$ & $\mathrm{IC}$ & $\mathrm{X}$ \\
$|Z|>\mathrm{UCL}$ & $\mathrm{T}$ & $\mathrm{XT}$ \\
\hline
\end{tabular}

simultaneous shifts in shapel parameter of the beta and shape parameter of unit gamma distribution is considered in Table S1. Similarly, for Case 1(b), a shift in shape 2 parameter of the beta distribution, keeping IC the rate parameter of the unit gamma distribution is considered in Table S2. Shift in the rate parameter of the unit gamma distribution, keeping IC the shape 2 parameter of the beta distribution, is considered in Table S3. Finally, simultaneous shifts in both the parameters are considered in Table S4.

To be specific, we considered the shift size $\Delta \in(1,1.10,1.20,1.30,1.40,1.90,0.80,0.70,0.60,0.50)$. The shifts are considered in the percentage form, that is, the value of $\Delta=0.90$ means that there is a $10 \%$ decrease in the parameter value, whereas the value $\Delta=1.10$ means that the value of the parameter is increased by $10 \%$. The value $\Delta=1$ corresponds to no shift in the parameter value. The IC values of mean and variance of plotting statistic M, i.e., EM and VM are also given in the tables. The analysis of the proposed scheme is conducted under the following general conditions.

For all of the cases given below, we have $\mu=0.20$

$$
\begin{aligned}
& \text { Case 1. } \tau=155 \text { and } \phi=290 \Rightarrow \delta_{0}=\mu \times \phi=58 \text {, } \\
& \gamma_{0}=(1-\mu) \phi=232 \text {, } \\
& \theta_{0}=\left(\mu^{1 / \tau} /\left(1-\mu^{1 / \tau}\right)\right)=95.80 \text {. } \\
& \text { Case 2. } \tau=96 \text { and } \phi=148 \Rightarrow \delta_{0}=\mu \times \phi=29.6 \text {, } \\
& \gamma_{0}=(1-\mu) \phi=118.4 \text {, } \\
& \tau_{0}=96 \text {, } \\
& \text { and } \\
& \theta_{0}=\left(\mu^{1 / \tau} /\left(1-\mu^{1 / \tau}\right)\right)=59.15 \text {. } \\
& \text { Case 3. } \tau=51 \text { and } \phi=80 \Rightarrow \delta_{0}=\mu \times \phi=16 \text {, } \\
& \gamma_{0}=(1-\mu) \phi=64 \\
& \theta_{0}=\left(\mu^{1 / \tau} /\left(1-\mu^{1 / \tau}\right)\right)=31.19 \text {. } \\
& \text { Case 4. } \tau=20 \text { and } \\
& \gamma_{0}=(1-\mu) \phi=24.8 \text {, } \\
& \theta_{0}=\left(\mu^{1 / \tau} /\left(1-\mu^{1 / \tau}\right)\right)=11.93 \text {. } \\
& \tau_{0}=51 \text {, } \\
& \text { and } \\
& \phi=31 \Rightarrow \delta_{0}=\mu \times \phi=6.2 \text {, } \\
& \tau_{0}=20 \text {, } \\
& \text { and }
\end{aligned}
$$

$\mathrm{ARL}_{0}$ is fixed at 370 and 500 to obtain L. The results are obtained using $\mathrm{R}$ software ( $\mathrm{R}$ version 4.0.0, 2020-04-24).

4.1. Results and Discussion. The analysis listed in Tables 3, 4 and Table $S 1$ is conducted to assess the performance of the proposed control chart under Case 1(a). More specifically, we have considered pure shifts in shape 1 parameter of $\mathrm{T}$ in Table 3. For the set of parameters given in Case 1, when $\lambda_{e}=0.05$ and $\mathrm{ARL}_{0}=370$, the ARL is observed to reduce by $94.46 \%, 97.62 \%, 98.45 \%$, and $98.82 \%$ with respect to the nominal ARL value when the shape 1 parameter of $\mathrm{T}$ is increased by $10 \%, 20 \%, 30 \%$, and $40 \%$, respectively, and reduced by $94.73 \%, 97.83 \%, 98.64 \%, 99.00 \%$, and $99.20 \%$ when the shape 1 parameter of $\mathrm{T}$ is increased by $10 \%, 20 \%$, $30 \%, 40 \%$, and $50 \%$, respectively. For the value of $\lambda_{e}=0.10$, the ARL is observed to reduce by $94.36 \%, 97.92 \%, 98.70 \%$, and $99.03 \%$ and by $94.69 \%, 98.14 \%, 98.87 \%, 99.18 \%$, and $99.37 \%$ for the same size of upward (UW) and downward (DW) shifts, respectively. Finally, for $\lambda_{e}=0.15$, the ARL is reduced by $93.86 \%, 98.02 \%, 98.82 \%$, and $99.13 \%$ and by $94.13 \%, 98.25 \%, 98.98 \%, 99.28 \%$, and $99.43 \%$, respectively, for the same amount of the UW and DW shifts in the shape1 parameter of $\mathrm{T}$.

Assuming $\mathrm{ARL}_{0}=500$ with $\lambda_{e}=0.05$, the ARL is reduced by $95.61 \%, 98.15 \%, 98.80 \%$, and $99.10 \%$ when there are pure UW shifts of size $10 \%, 20 \%, 30 \%$, and $40 \%$ in shape 1 parameter of $\mathrm{T}$ and reduced by $95.88 \%, 98.33 \%, 98.95 \%$, $99.23 \%$, and $99.39 \%$ in the case of pure DW shifts of size $10 \%, 20 \%, 30 \%, 40 \%$, and $50 \%$, respectively. A similar pattern is observed for $\lambda_{e}=0.10$ and 0.15 .

For the parameters given in Case 2, when $\lambda_{e}=0.05$ and $\mathrm{ARL}_{0}=370$, the reduction in the ARL is by $90.96 \%, 96.45 \%$, $97.77 \%$, and $98.34 \%$ for $10 \%, 20 \%, 30 \%$, and $40 \%$ for the UW shifts and by $91.50 \%, 96.81 \%, 98.07 \%, 98.61 \%$, and $98.92 \%$ for $10 \%, 20 \%, 30 \%, 40 \%$, and $50 \%$ DW shifts, respectively. Similarly, for $\lambda_{e}=0.10$, the ARL is reduced by $90.12 \%$, $96.69 \%, 98.06 \%$, and $98.60 \%$ and by $90.36 \%, 97.09 \%, 98.36 \%$, $98.86 \%$, and $99.11 \%$, respectively, for the same amount of the UW and DW shifts. A similar pattern can be observed for $\lambda_{e}=0.15$. In the case of $\mathrm{ARL}_{0}=500$, the behavior of the ARL is observed very similar as $\mathrm{ARL}_{0}=370$.

The behavior of the ARL for case 3 and case 4 can be interpreted in a similar fashion. Table 4 considers pure shifts in the shape parameter of $X$. For the set of parameters given in case 1 , when $\lambda_{e}=0.05$, the ARL is reduced by $97.24 \%, 98.62 \%, 99.01 \%$, and $99.18 \%$ with respect to the nominal value of $\mathrm{ARL}_{0}=370$ when $\Delta_{\tau} \in(1.10,1.20,1.30,1.40)$, respectively. Furthermore, when $\Delta_{\tau} \in(0.90,0.80,0.70$, $0.60,0.50)$, the ARL is reduced by $97.71 \%, 98.98 \%, 99.35 \%$, $99.49 \%$, and $99.64 \%$, respectively. For $\lambda_{e}=0.10$ and 0.15 , a similar reduction in the ARL is observed for both 370 and 500 nominal values of the ARL. Similarly, for case 2 of the parameters, the ARL is reduced by $97.54 \%, 98.86 \%, 99.18 \%$, and $99.36 \%$ when there is the UW shift of the size mentioned above and by $98.00 \%, 99.17 \%, 99.46 \%, 99.61 \%$, and $99.72 \%$ when there is a DW shift of similar size as mentioned above. Finally, for $\lambda_{e}=0.15$, the ARL is reduced by $97.59 \%, 98.97 \%$, $99.28 \%$, and $99.43 \%$ for the UW shifts and by $98.10 \%$, $99.25 \%, 99.51 \%, 99.68 \%$, and $99.73 \%$ for the DW shifts in the shape parameter of $\mathrm{X}$. The interpretation of the remaining cases can be done similarly. According to the ARL criteria, the chart based on pure shift in the shape parameter of $X$ performs better than the one based on pure shift in shape 1 parameter of $\mathrm{T}$.

For simultaneous shifts in the two parameters of $\mathrm{X}$ and $\mathrm{T}$, Table S1, the similar degenerating pattern of ARL is observed for increasing UW and DW shifts. Specifically, when $\Delta_{\tau}=1.10$ and $\Delta_{\delta} \in(1.10,1.20,1.30,1.40)$ in Case 1 , i.e., simultaneous $10 \% \mathrm{UW}$ shift in shape parameter of $\mathrm{X}$ and $10 \%, 20 \%, 30 \%$, and $40 \%$ UW shift in the shape 1 parameter of $\mathrm{T}$, the ARL is reduced by $97.35 \%, 97.90 \%, 98.47 \%$, and $98.83 \%$, respectively. On the other hand, when 


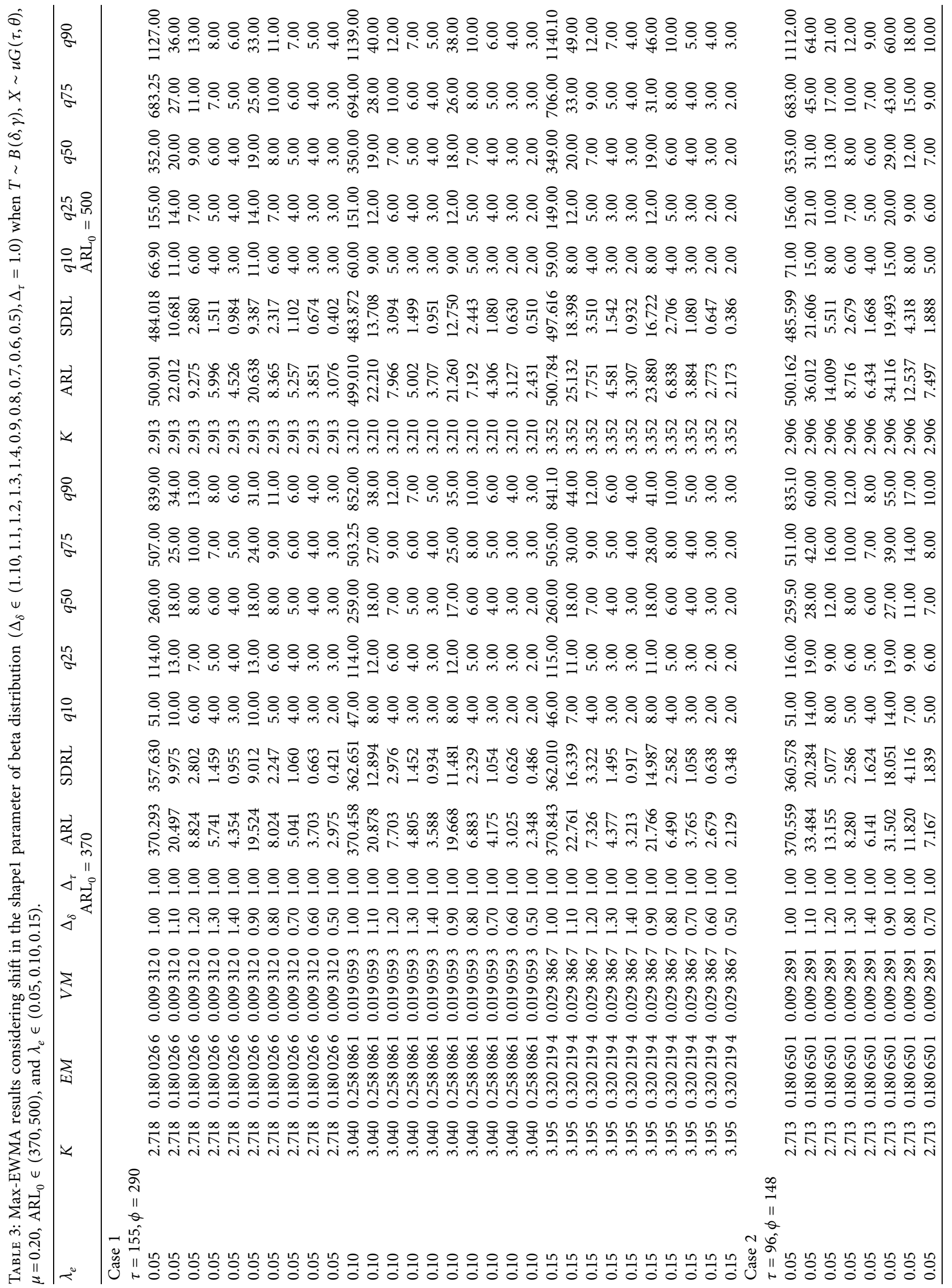




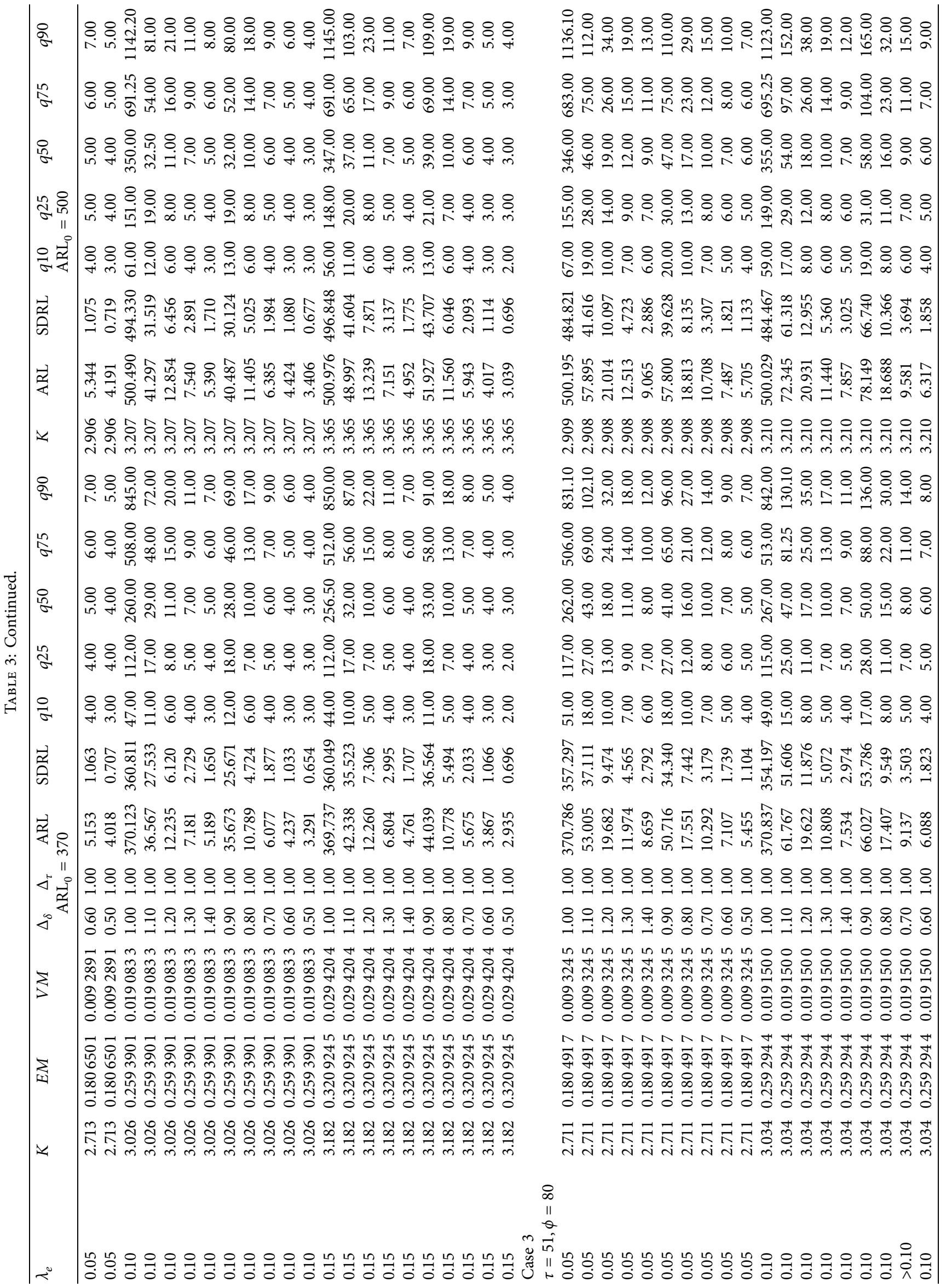




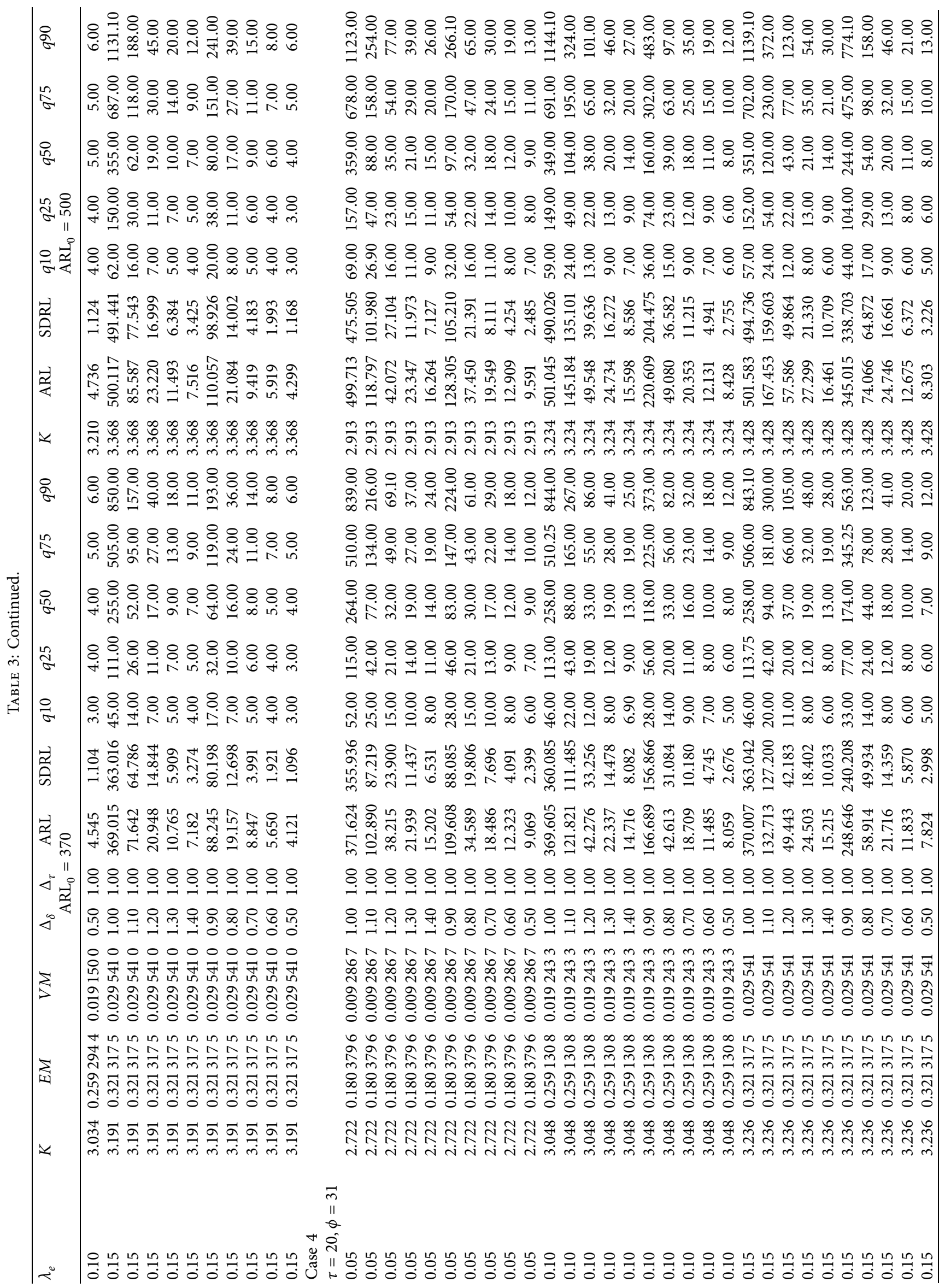




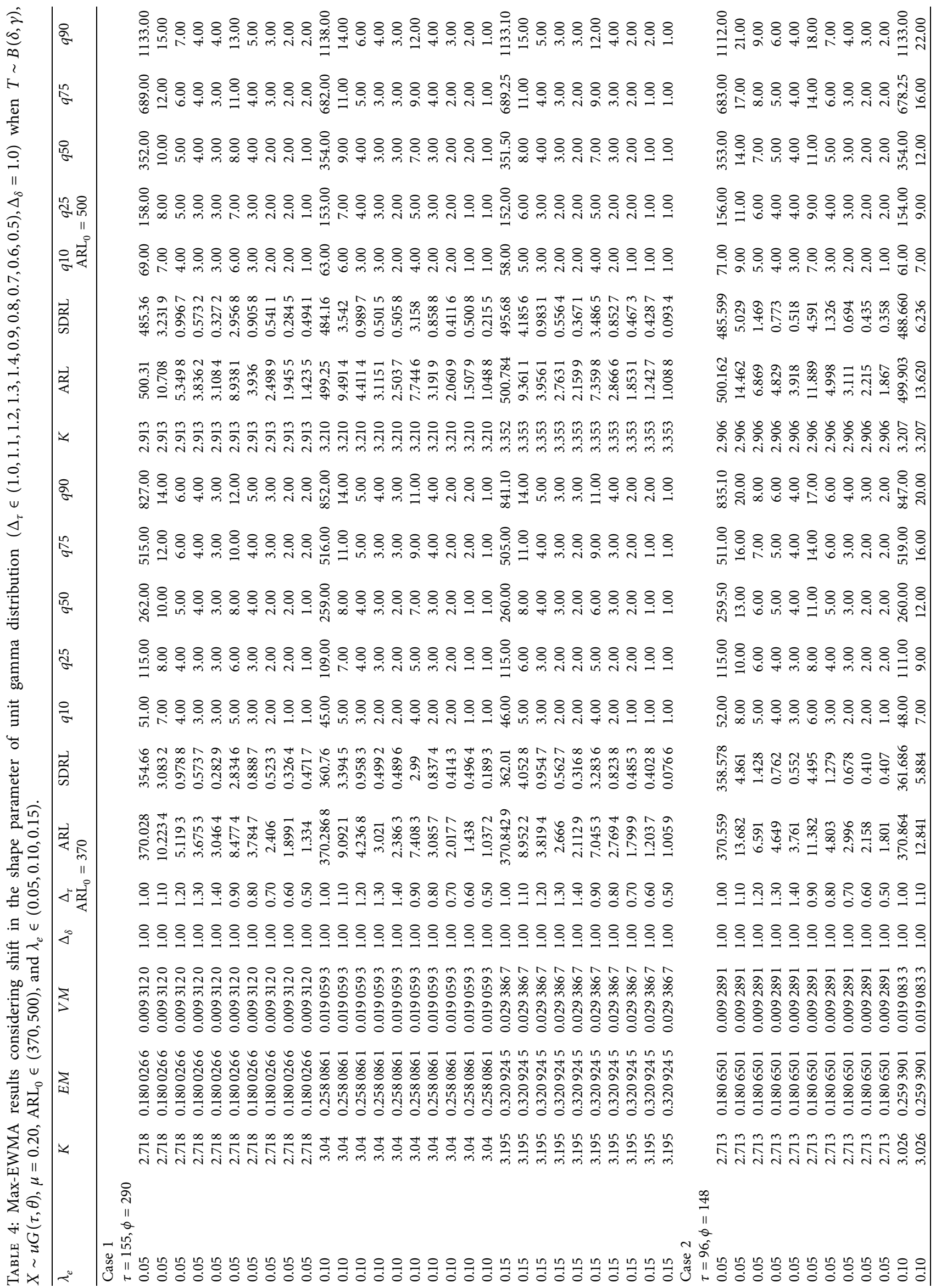




\begin{tabular}{|c|c|c|}
\hline \& & 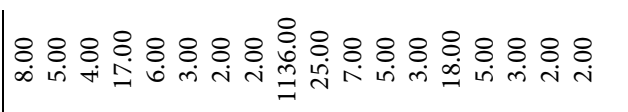 & 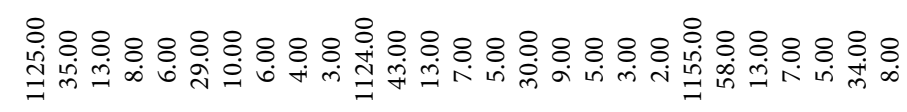 \\
\hline$\frac{\mathfrak{L}}{\alpha}$ & 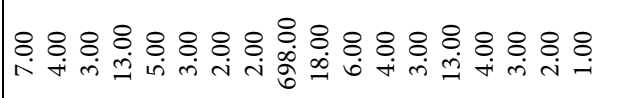 & 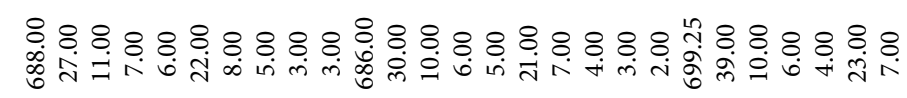 \\
\hline in & 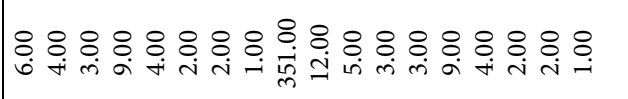 & 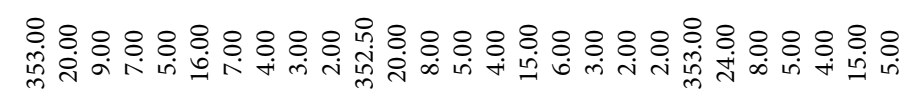 \\
\hline 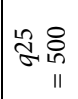 & \& \& \& \& \& \& \& \& \& \& \& \& \& \& \& \& \& \& & 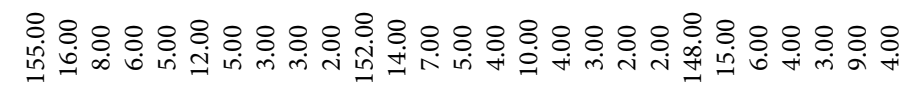 \\
\hline 号迸 & \& \& \& \& \& \& \& \& \& \& \& \& \& \& \& \& \& \& \& \& 8 & 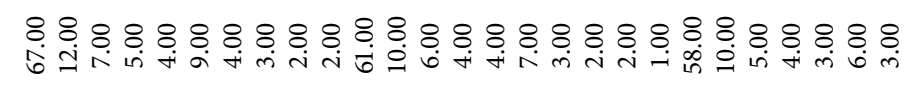 \\
\hline 究 & 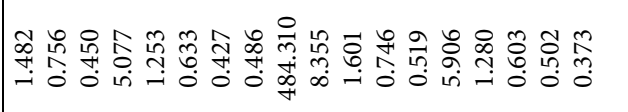 & 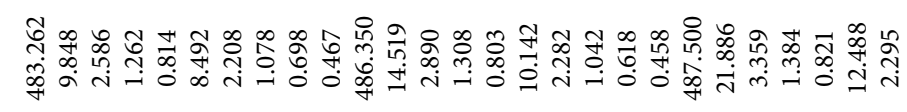 \\
\hline $\overrightarrow{\mathrm{e}}$ & 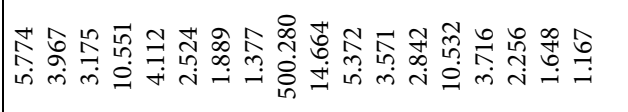 & 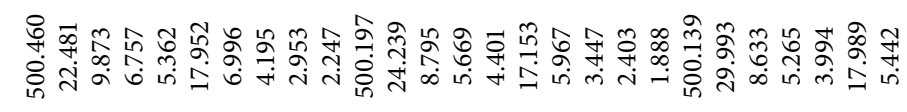 \\
\hline 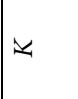 & 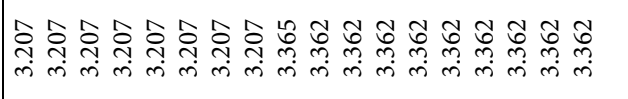 & 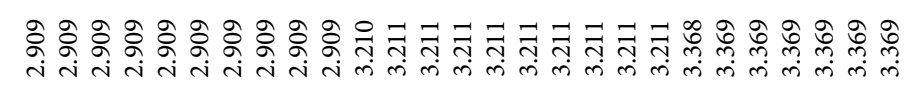 \\
\hline \&े & 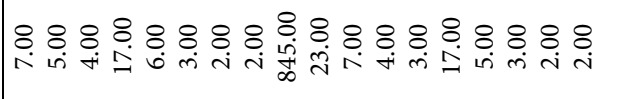 & 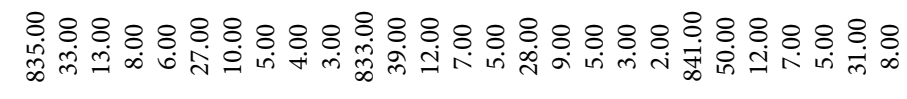 \\
\hline$\stackrel{n}{\text { of }}$ & 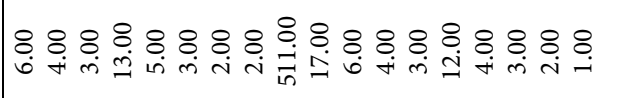 & 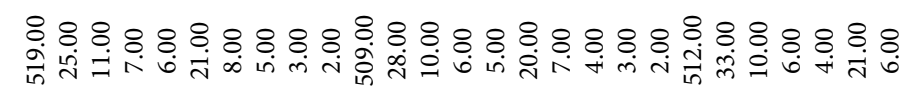 \\
\hline 염 & 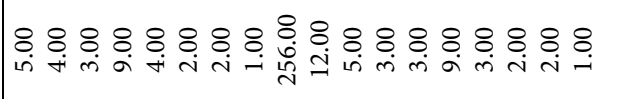 & 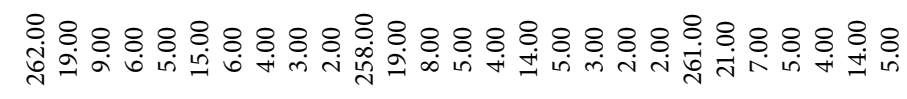 \\
\hline$\stackrel{n}{2}$ & 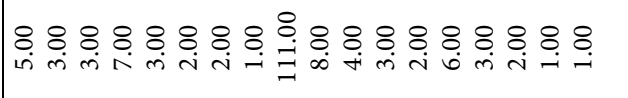 & 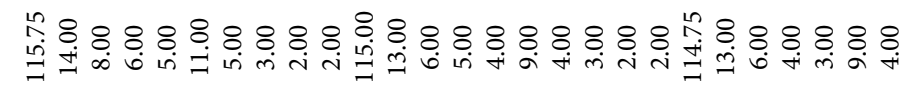 \\
\hline$\stackrel{一}{\sigma}$ & 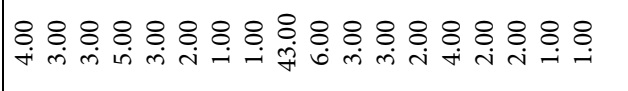 & 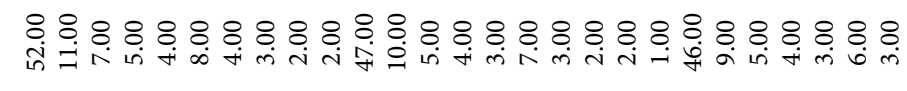 \\
\hline ڤี & 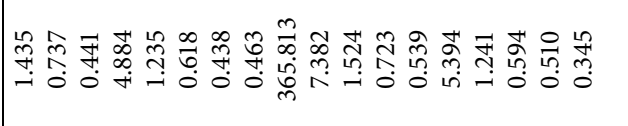 & 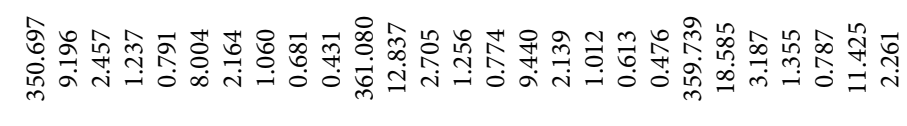 \\
\hline 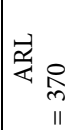 & 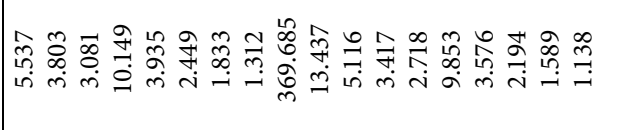 & 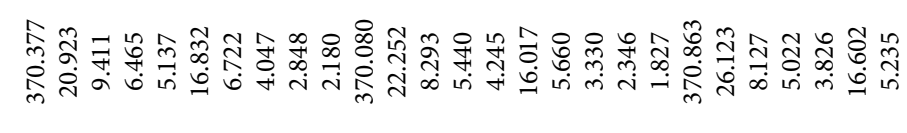 \\
\hline$\triangleleft \stackrel{0}{\frac{3}{4}}$ & 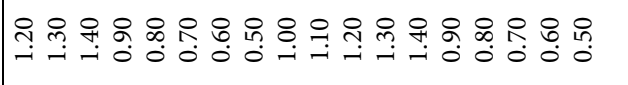 & 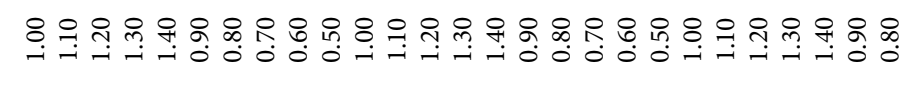 \\
\hline$\triangleleft$ & \& \& \& \& \& \& \& \& \& \& \& \& \& \& \& \& \& \& \& \& \& & 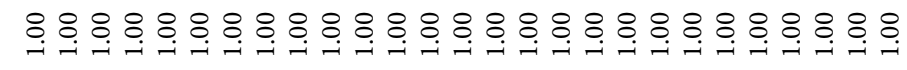 \\
\hline$\sum$ & 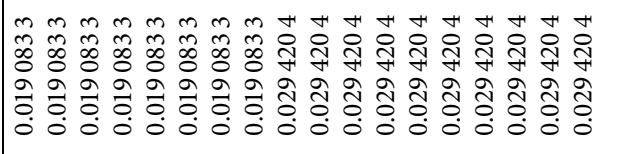 & 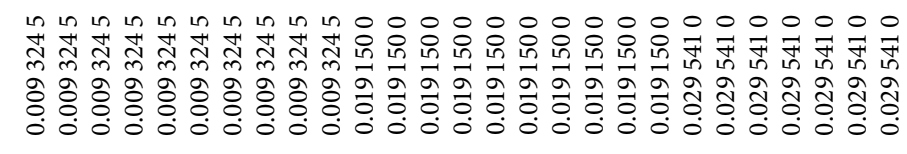 \\
\hline$\sum$ & 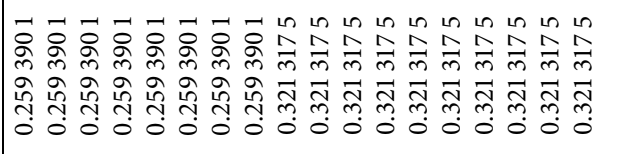 & 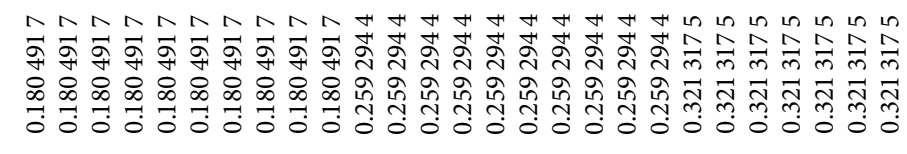 \\
\hline$\approx$ & 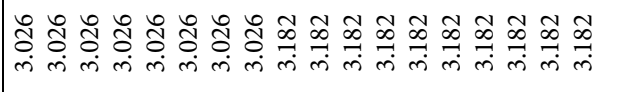 & 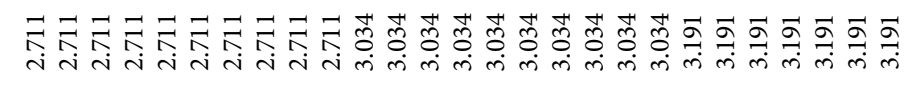 \\
\hline & 00 & \\
\hline
\end{tabular}




\begin{tabular}{|c|c|c|}
\hline \&े & $\underset{i}{8} \underset{i}{8} \underset{i}{8}$ & 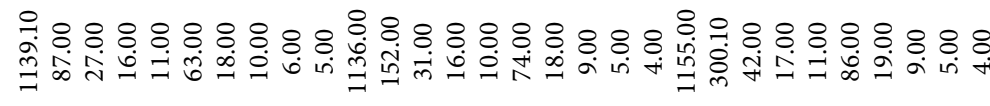 \\
\hline ô & $\underset{+i}{8} 8$ & 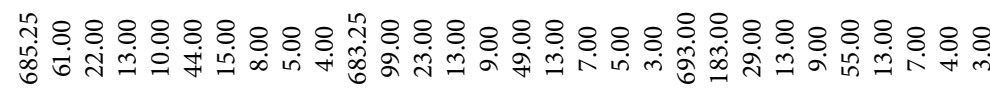 \\
\hline 品 & $\stackrel{8}{8} \underset{i}{8} \stackrel{8}{i}$ & 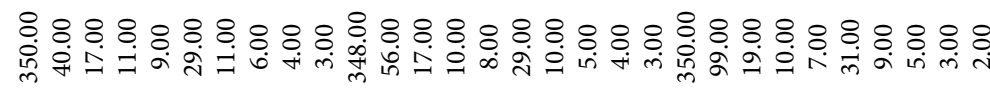 \\
\hline 요용 & 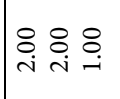 & 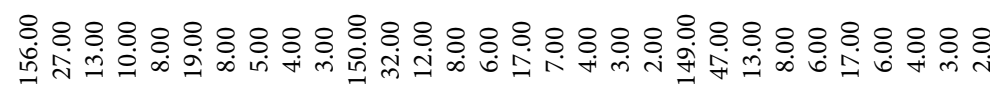 \\
\hline$\frac{\mathrm{d}}{\mathrm{a}}$ & $\underset{i}{8} \stackrel{8}{8} \underset{i}{8}$ & 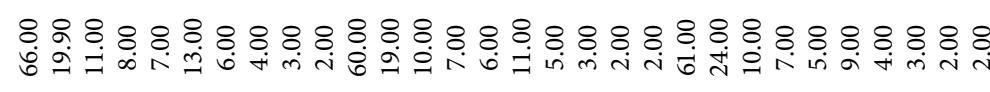 \\
\hline 究 & 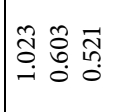 & 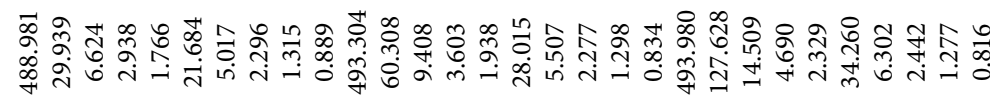 \\
\hline $\overrightarrow{\mathrm{s}}$ & 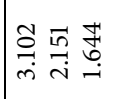 & 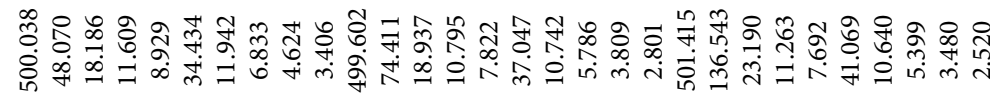 \\
\hline 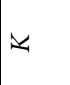 & 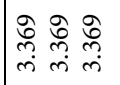 & 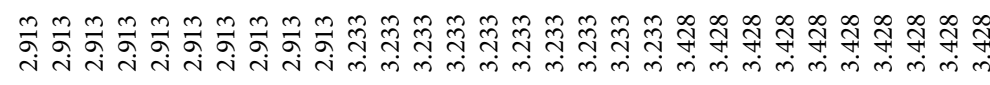 \\
\hline \&े & 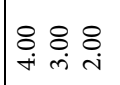 & 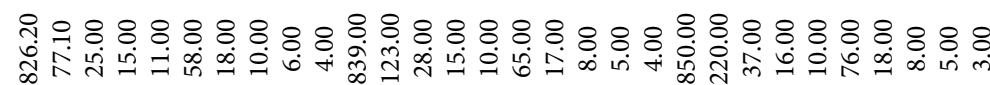 \\
\hline$\stackrel{n}{o r}$ & $\underset{+i}{8} \underset{i}{8} \stackrel{8}{i}$ & 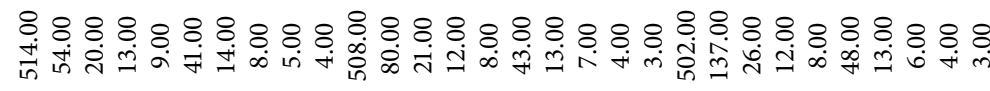 \\
\hline 总 & 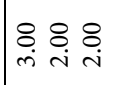 & 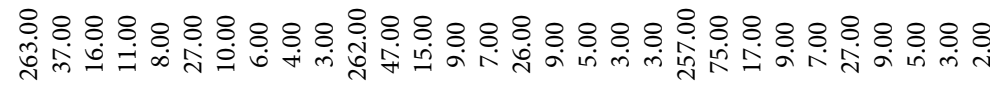 \\
\hline 䟤 & 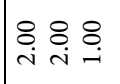 & 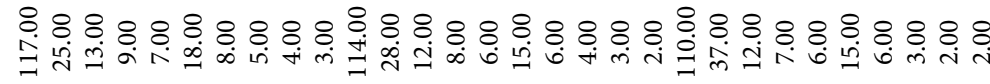 \\
\hline$\%$ & 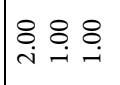 & 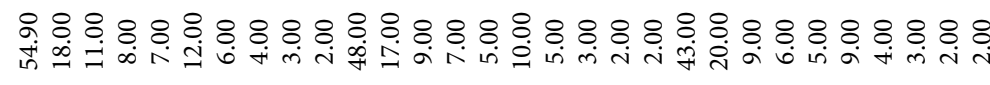 \\
\hline कि & 它 & 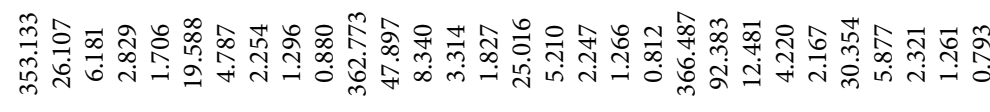 \\
\hline 란 & 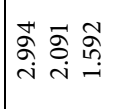 & 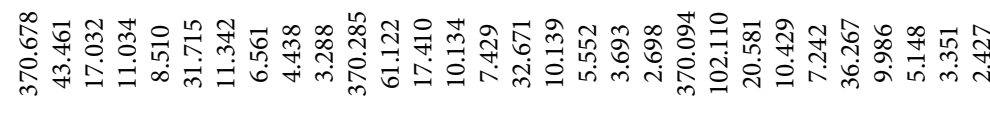 \\
\hline 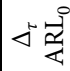 & R $\begin{array}{lll}R & 0 \\
0 & 0 & 0 \\
0 & 0 & 0 \\
0\end{array}$ & 进억 억 \\
\hline$\triangleleft^{\infty}$ & 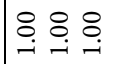 & 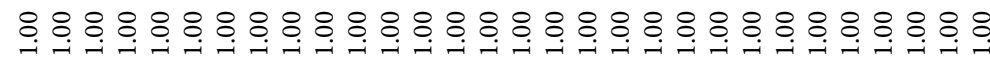 \\
\hline$\sum_{S}$ & 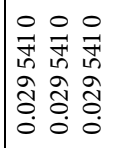 & 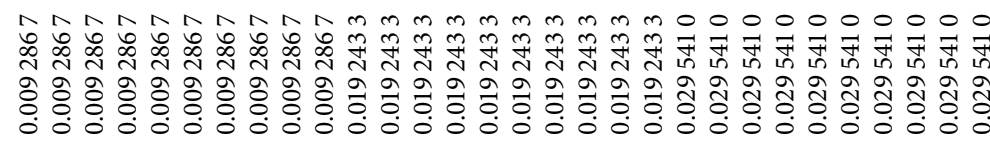 \\
\hline 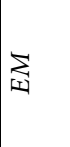 & 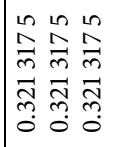 & 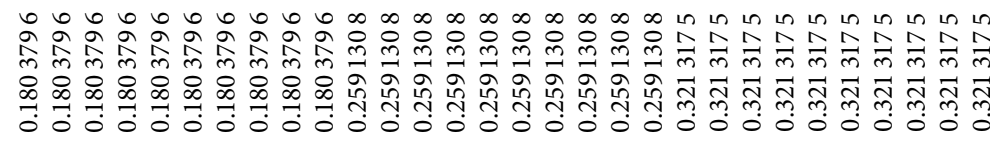 \\
\hline 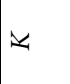 & $\overrightarrow{\vec{m}} \overrightarrow{\dot{m}} \overrightarrow{\vec{m}}$ & 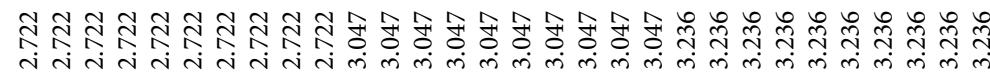 \\
\hline & $\operatorname{lng}_{0}^{\circ}: \frac{1}{0}$ & \\
\hline
\end{tabular}


$\Delta_{\delta} \in(0.90,0.80,0.70,0.60,0.50)$, the ARL is reduced by $97.36 \%, 98.00 \%, 98.65 \%, 99.00 \%$, and $99.20 \%$ for the same amount of shift in the shape parameter of X. In addition, when a UW shift in the shape parameter of $\mathrm{X}$ is increased to $20 \%$, the ARL is reduced by $98.62 \%, 98.64 \%, 98.73 \%$, and $98.89 \%$ and by $98.62 \%, 98.64 \%, 98.77 \%, 99.01 \%$, and $99.20 \%$ for similar UW and DW shifts in the shape 1 parameter of T as given above. The same degeneration in the ARL values is observed for various values of smoothing parameter. However, it is worth noting that the proposed chart performs exceptionally well for the Case 1 and Case 2 of the parameters, but as we move down the table to Case 3 and Case 4, the performance deteriorated, especially in the case of smaller UW and DW shifts whether pure or simultaneous. For example, when $\lambda_{e}=0.15$ and $\mathrm{ARL}_{0}=500$ in Case 4 of Table 3, the ARL reduced only by $66.62 \%$ for $10 \%$ UW and only by $31.21 \%$ for $10 \%$ DW shift which is substantially lower compared to Case 1 for similar value of $\mathrm{ARL}_{0}$ and $\lambda_{e}$. When there is a simultaneous shift of size $\left(\Delta_{\delta}, \Delta_{\tau}\right)=(1.10,1.10)$ in Case 4 of Table $\mathrm{S} 1$, for $\mathrm{ARL}_{0}=370$ and $\lambda_{e}=0.15$, the ARL reduced only by $81.02 \%$, whereas in Case 1 , the reduction in ARL is by $97.76 \%$ for a similar shift size and smoothing parameter. The standard deviation of ARL (SDRL) has the same decreasing pattern for all of the cases. Thus, it is concluded that the ARL and SDRL decrease by increasing the UW or DW shifts.

The percentile analysis shows that, in most cases, the ARL is greater than the median (q50) but less than the $75^{\text {th }}$ percentile (q75) suggesting that the run length distribution is positively skewed. For example, when $\Delta_{\delta}=1.10, \lambda_{e}=0.05$, and $\mathrm{ARL}_{0}=370$ in Case 1 of Table 3, the ARL is 20.497, the median is 18 , and q75 is 25 . Interestingly, in some cases the ARL is slightly less than the median which means that the distribution is slightly negatively skewed. For example, in case 1 of Table 3 assuming $\Delta_{\delta}=1.30$ and $\lambda_{e}=0.05$, the ARL is 5.741 and the median is 6; however, in Table 4, when $\Delta_{\tau}=$ 1.30 and $\lambda_{e}=0.05$ in case 1 , the ARL is 3.675 and the median is 4 . Thus, the proposed chart is more sensitive to small shifts $\left(\Delta_{\delta}, \Delta_{\tau} \in(1.10,0.90)\right)$ for pure or simultaneous shifts with $\lambda_{e}=0.05$. It is to be noted that the sensitivity to moderate shifts increased when $\lambda_{e}$ increased. For example, in case 1 of Table 3, when $\Delta_{\delta}=1.10,0.90$ (small shifts) and $\lambda_{e}=0.05$, the $\mathrm{ARL}_{1}$ is 20.497 and 19.524, while the corresponding $\mathrm{ARL}_{1}$ values with $\lambda_{e}=0.15$ are 22.761 and 21.766 , respectively. On the other hand, when $\Delta_{\delta}=1.40$ (a large shift), the $A R L_{1}$ value for $\lambda_{e} \in(0.05,0.10,0.15)$ is $(4.354,3.588,3.213)$, respectively. For a simultaneous shift of size $\left(\Delta_{\delta}, \Delta_{\tau}\right)=(1.10,1.10)$ as reported in case 4 in Table S1, the $\mathrm{ARL}_{1}$ is 38.66 for $\lambda_{e}=0.05$ and 70.28 for $\lambda_{e}=0.15$. However, this does not hold for all cases. For example, in Case 1 of Table S1 when $\lambda_{e} \in(0.05,0.15)$ the $\mathrm{ARL}_{1}$ is $(9.83,8.28)$ for $10 \%$ simultaneous UW shift in both parameters. It is worth noting that the behavior of the ARL does not conform to the traditional fashion, that is, small smoothing parameters are suitable for small shifts and vice versa. As this discrepancy exists only for a few $\mathrm{ARL}_{1}$ values, it is safe to say that smaller values of $\lambda_{e}$ are suitable for detecting smaller shifts and larger values are suitable to detect larger shifts.
In Table S2, we have considered pure shift in the shape 2 parameter of $\mathrm{T}$, whereas in Table S3, pure shift in the rate parameter of $X$ is considered. Finally, a simultaneous shift in shape 2 parameter of $\mathrm{T}$ and rate parameter of $\mathrm{X}$ is considered in Table S4. From the tables, we observed a very similar degenerating pattern in the ARL as well as in the SDRL as noted earlier. More specifically, in the case of pure shift, we can see that an UW shift in the shape 1 parameter of $\mathrm{T}$ is detected more quickly as compared to the UW shift in the shape 2 parameter of T. On the other hand, a DW shift in shape 2 is detected more quickly. For example, when $\lambda_{e}=0.05, A R L_{0}=370$, and $\Delta_{\delta} \in(1.30,1.40)$ in Case 1 of Table $3, \mathrm{ARL}_{1}=(5.741,4.354)$, and when $\Delta_{\gamma}=(1.30,1.40)$ under similar settings in Table $4, A R L_{1}=(6.735,5.278)$. On the other hand, when $\Delta_{\delta} \in(0.90,0.80)$ in Case $1, \mathrm{ARL}_{1}=$ $(19.524,8.024)$ and $A R L_{1}=(17.912,6.971)$ when $\Delta_{y}=(0.90,0.80)$ in Case 1. While comparing the efficiency of the proposed chart for detecting pure shifts in shape and rate parameter of $\mathrm{X}$, we can see that an UW shift in the rate parameter is detected more quickly, whereas a DW shift in the shape parameter of $\mathrm{X}$ is detected more quickly. The performance of the chart for simultaneous shifts in Table S1 is more or less the same as given in Table S4.

\section{Real Data Application}

In this section, the proposed methodology is applied to a real data set taken from a disaster management department that controls fire [20]. Controlling fire in an efficient manner leaves a positive impact than an uncontrolled occurrence of fire that may cause severe destruction. A disaster management department always tries to monitor the damages inflicted by the fire outbreaks to minimize the future losses. There are 25 observations in the data set including two variables: the amount of losses (in 1000\$) and the time interval (in days) between the successive fire outbreaks. Both of these variables are transformed into proportion. The magnitude (X) represents the proportion of losses, whereas the TBE $(\mathrm{T})$ represents the proportion of days between the successive fire outbreaks. Our interest here is to detect an increase in the amount of losses and/or decrease in the time between the fire outbreaks. The variable $\mathrm{T}$ is fitted to beta distribution, and variable $\mathrm{X}$ is fitted to unit gamma distribution. To estimate the parameters, we have used the maximum likelihood approach, and all the calculations are done using the statistical software R. Apart from the first 25 observations which are used as the phase I sample, we have further simulated 25 values as the phase II observations by introducing shifts of $\Delta_{\delta}=0.80$ and $\Delta_{\tau}=0.80$ to exhibit the application of the proposed charting scheme. Empirical mean and variance of the plotting statistic are 0.2545 and 0.0214 , respectively. Using $\lambda_{e}=0.15$ and $L=1.90$, we obtained $\mathrm{UCL}=0.5325$ with $\mathrm{ARL}_{0}=25$. The maximum likelihood estimates of the parameters along with their standard errors (in parentheses), the Akaike information criterion (AIC) and Bayesian information criterion (BIC), for the 25 IC observations are presented in Table 5. Figure 1 presents the Max-EWMA control chart constructed for the data set 
TABle 5: Parameter estimates with standard errors (in parentheses) and goodness-of-fit statistics for the proportion of days between successive fire outbreaks and proportion of losses.

\begin{tabular}{|c|c|c|c|c|c|}
\hline \multicolumn{2}{|l|}{ Distribution } & \multicolumn{2}{|c|}{ ML estimates } & \multirow{3}{*}{$\frac{\text { AIC }}{-106.8893}$} & \multirow{3}{*}{$\frac{\text { BIC }}{-104.4516}$} \\
\hline Ret? & $\widehat{\mu}$ & 0.0402 & $(0.0067)$ & & \\
\hline Beta & $\widehat{\phi}$ & 21.1089 & $(2.0994)$ & & \\
\hline Unit gamma & $\begin{array}{l}\widehat{\mu} \\
\widehat{\tau}\end{array}$ & $\begin{array}{c}0.9222 \\
39.7338\end{array}$ & $\begin{array}{c}(0.0197) \\
(10.8423)\end{array}$ & -124.5977 & -122.1600 \\
\hline
\end{tabular}

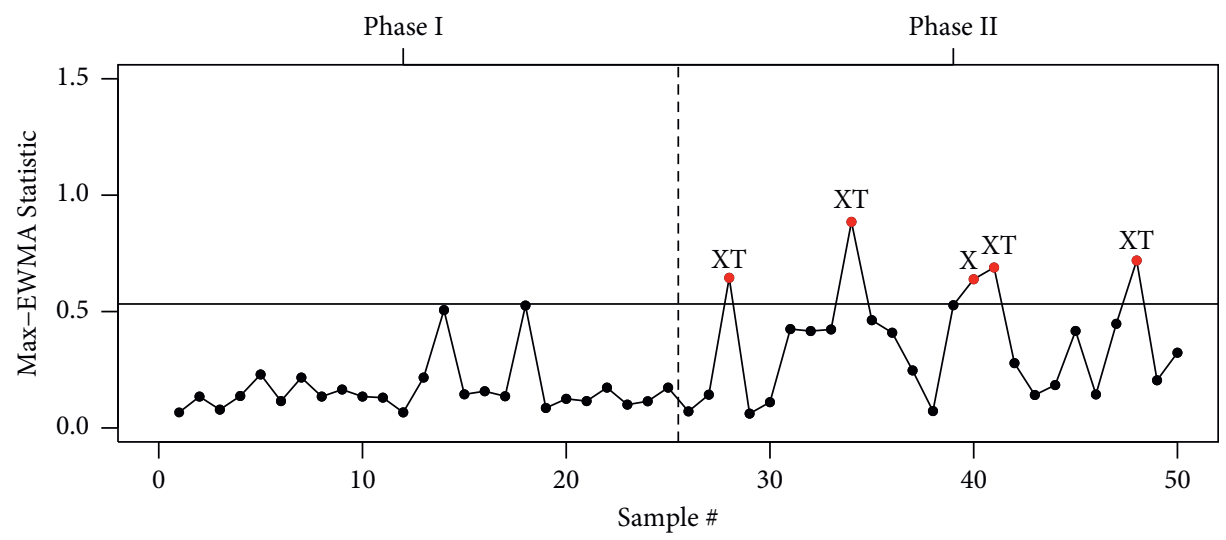

Figure 1: Max-EWMA chart for monitoring the fire outbreaks considering simultaneous shift in shape 1 parameter of beta distribution and shape parameter of unit gamma distribution.

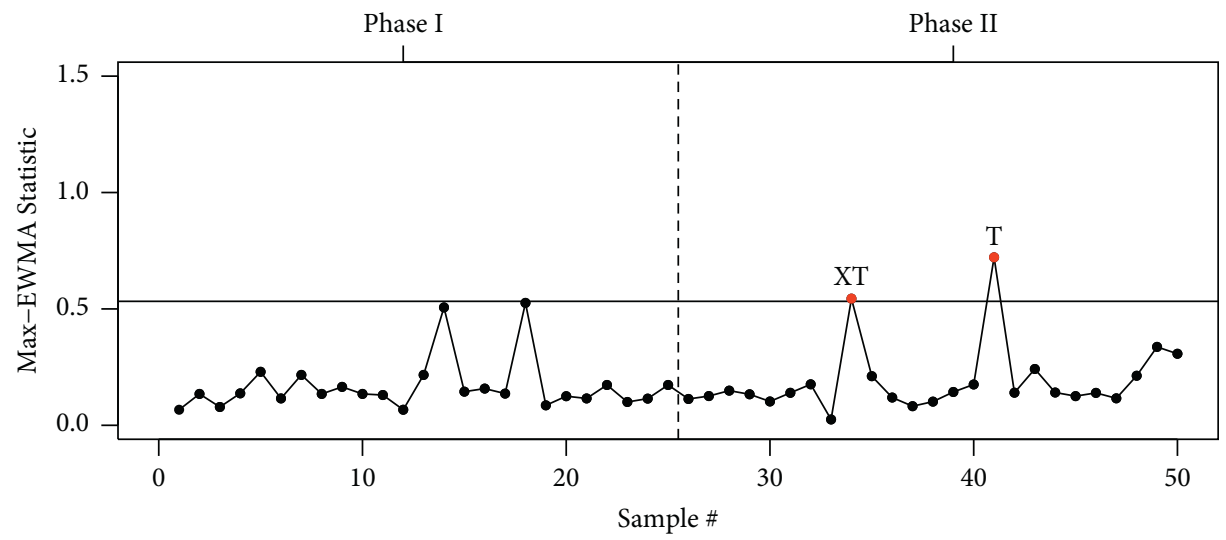

FIgURE 2: Max-EWMA chart for monitoring the fire outbreaks considering the pure shift in the shape 1 parameter of beta distribution.

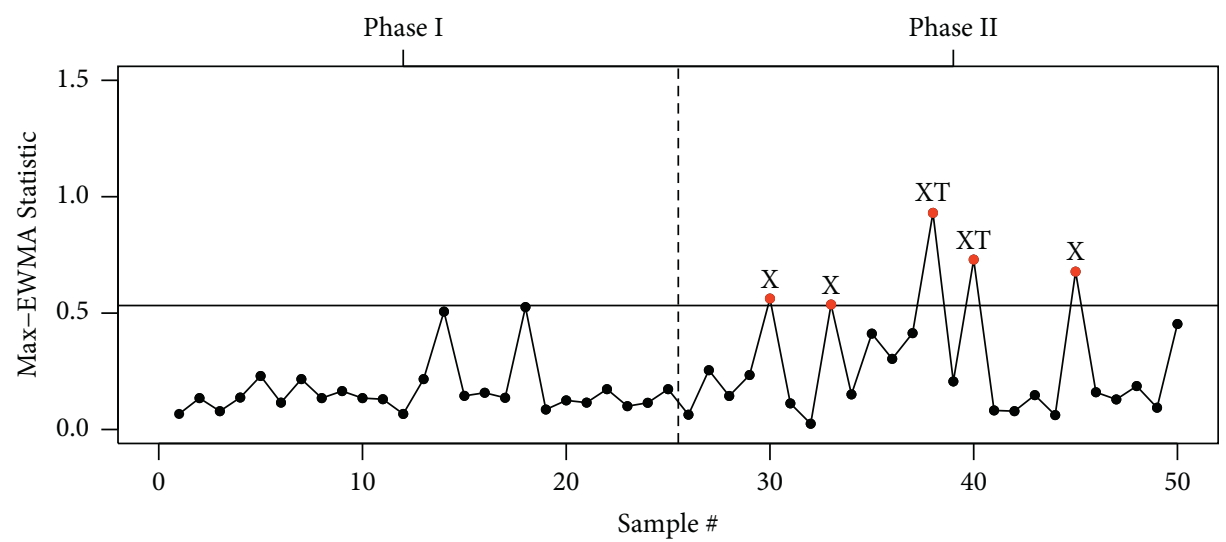

Figure 3: Max-EWMA chart for monitoring the fire outbreaks considering the pure shift in the shape parameter of unit gamma distribution. 
(phase I and phase II). There appears no signal in phase I data as the chart gives no OOC signal; however, the first OOC signal is observed at the $28^{\text {th }}$ sample. When a shift in the fire outbreaks, such as this one, is observed, the disaster management department can look into the reasons behind the phenomenon and try to eliminate them.

Furthermore, we have constructed the Max-EWMA chart for $80 \%$ pure shift in the parameters. Specifically, when there is a pure shift in the shape 1 parameter of T, Figure 2 shows that the first OOC signal is triggered at the $34^{\text {th }}$ sample. On the other hand, if there is a pure shift in the shape parameter of the unit gamma distribution, Figure 3, the first OOC signal is triggered at the $30^{\text {th }}$ sample. These charts indicate that the simultaneous shift is detected more quickly as compared to the pure shift. The OOC points are labelled with respective labels to identify the signals.

\section{Conclusion}

With the advancement in technology, many human centric jobs have been taken over by machines and it is imperative to monitor the output of these machines to assess whether the performance level is up to the expectations or not. The SPC provides tools and techniques which enabled us to distinguish between assignable and natural causes in a process. A number of techniques have already been developed to monitor processes with different assumptions, distributional forms, and range of data involved; however, there are very few studies related to unit interval data. Therefore, motivated by the importance of unit interval data, we introduced a Max-EWMA chart for simultaneous monitoring of the magnitude and TBE of an event under the assumption that TBE follows the beta distribution and magnitude of the event follows unit gamma distribution. In order to evaluate the performance of the proposed chart, the most frequently used criterion, i.e., the ARL has been used. The results indicate that the overall performance of the proposed chart is substantially good for small to medium-sized shifts. The current study also emphasizes on the fact that simultaneous monitoring of magnitude and frequency is more efficient as compared to individual monitoring of both these characteristics. Finally, the charting procedure is applied to a real life data set. In the future, the current work can be extended by considering some new distributions dealing with unit interval data. Furthermore, the effect of parameter estimation on phase I and phase II may be studied in detail. Also, a recent idea of neutrosophic statistics [28] can be utilized to extend the present work.

\section{Data Availability}

The dataset used in the analysis is available upon request from the first author.

\section{Conflicts of Interest}

The authors declare that they have no conflicts of interest.

\section{Supplementary Materials}

The supplementary PDF file contains additional tables for simulation studies. . (Supplementary Materials)

\section{References}

[1] W. H. Woodall, "Control charts based on attribute data: bibliography and review," Journal of Quality Technology, vol. 29, no. 2, pp. 172-183, 1997.

[2] L. Lee Ho and R. C. Quinino, "An attribute control chart for monitoring the variability of a process," International Journal of Production Economics, vol. 145, no. 1, pp. 263-267, 2013.

[3] L. Lee Ho, F. H. Fernandes, and M. Bourguignon, "Control charts to monitor rates and proportions," Quality and Reliability Engineering International, vol. 35, no. 1, pp. 74-83, 2019.

[4] Z. Qiu, P. X. K. Song, and M. Tan, "Simplex mixed-effects models for longitudinal proportional data," Scandinavian Journal of Statistics, vol. 35, no. 4, pp. 577-596, 2008.

[5] Â. M. O. Sant'Anna and C. S. ten Caten, "Beta control charts for monitoring fraction data," Expert Systems with Applications, vol. 39, no. 11, pp. 10236-10243, 2012.

[6] L. M. D. A. Lima-Filho, T. L. Pereira, T. C. D. Souza, and F. M. Bayer, "Inflated beta control chart for monitoring double bounded processes," Computers \& Industrial Engineering, vol. 136, pp. 265-276, 2019.

[7] S. Dey, A. F. Menezes, and J. Mazucheli, "Comparison of estimation methods for unit-gamma distribution," Journal of Data Science, vol. 17, no. 4, pp. 768-801, 2019.

[8] A. Grassia, "On a family of distributions with argument between 0 and 1 obtained by transformation of the gamma and derived compound distributions," Australian Journal of Statistics, vol. 19, no. 2, pp. 108-114, 1977.

[9] M. V. Ratnaparkhl and J. E. Mosimann, "On the normality of transformed beta and unit- gamma random variables," Communications in Statistics-Theory and Methods, vol. 19, no. 10, pp. 3833-3854, 1990.

[10] P. R. Tadikamalla, "On a family of distributions obtained by the transformation of the gamma distribution," Journal of Statistical Computation and Simulation, vol. 13, no. 3-4, pp. 209-214, 1981.

[11] A. M. Mousa, A. A. El-Sheikh, and M. A. Abdel-Fattah, "A gamma regression for bounded continuous variables," $A d$ vances and Applications in Statistics, vol. 49, no. 4, pp. 305326, 2016.

[12] T. Goh, "A control chart for very high yield processes," Quality Assurance, vol. 13, no. 1, pp. 18-22, 1987.

[13] S. Ali, A. Pievatolo, and R. Göb, "An overview of control charts for high-quality processes," Quality and Reliability Engineering International, vol. 32, no. 7, pp. 2171-2189, 2016.

[14] S. Ali, "Time-between-events control charts for an exponentiated class of distributions of the renewal process," Quality and Reliability Engineering International, vol. 33, no. 8, pp. 2625-2651, 2017.

[15] M. Xie, T. N. Goh, and P. Ranjan, "Some effective control chart procedures for reliability monitoring," Reliability Engineering \& System Safety, vol. 77, no. 2, pp. 143-150, 2002.

[16] C. W. Zhang, M. Xie, J. Y. Liu, and T. N. Goh, "A control chart for the gamma distribution as a model of time between events," International Journal of Production Research, vol. 45, no. 23, pp. 5649-5666, 2007. 
[17] M. S. Shafae, R. M. Dickinson, W. H. Woodall, and J. A. Camelio, "Cumulative sum control charts for monitoring weibull-distributed time between events," Quality and Reliability Engineering International, vol. 31, no. 5, pp. 839-849, 2015.

[18] I. Shah, B. Iqbal, M. F. Akram, and S. Ali, "Unit Nadarajah and Haghighi distribution: properties and applications in quality control," Scientia Iranica, 2021.

[19] A. Talib, S. Ali, and I. Shah, "Max-EWMA chart for time and magnitude monitoring using exponentially modified Gaussian distribution," Quality and Reliability Engineering International, vol. 38, no. 2, pp. 1092-1111, 2021.

[20] Z. Wu, J. Jiao, and Z. He, "A single control chart for monitoring the frequency and magnitude of an event," International Journal of Production Economics, vol. 119, no. 1, pp. 24-33, 2009.

[21] Z. Wu, J. Jiao, and Z. He, "A control scheme for monitoring the frequency and magnitude of an event," International Journal of Production Research, vol. 47, no. 11, pp. 2887-2902, 2009.

[22] Y. Liu, Z. He, L. Shu, and Z. Wu, "Statistical computation and analyses for attribute events," Computational Statistics \& Data Analysis, vol. 53, no. 9, pp. 3412-3425, 2009.

[23] L. Qu, Z. Wu, M. B. C. Khoo, and L. J. Shu, "A new control chart for monitoring the event frequency and magnitude," European Journal of Industrial Engineering, vol. 8, no. 6, pp. 789-813, 2014.

[24] N. A. Adegoke, M. Riaz, R. A. Sanusi, A. N. H. Smith, and M. D. M. Pawley, "EWMA-type scheme for monitoring location parameter using auxiliary information," Computers \& Industrial Engineering, vol. 114, pp. 114-129, 2017.

[25] D. M. Hawkins and D. H. Olwell, Cumulative Sum Charts and Charting for Quality Improvement, Springer Science \& Business Media, Berlin, Germany, 2012.

[26] R. A. Sanusi, S. Y. Teh, and M. B. C. Khoo, "Simultaneous monitoring of magnitude and time-between-events data with a max-EWMA control chart," Computers \& Industrial Engineering, vol. 142, p. 106378, 2020.

[27] D. C. Montgomery, Introduction to Statistical Quality Control, John Wiley \& Sons, HOboken, NY, USA, 2007.

[28] M. Aslam, G. S. Rao, M. Saleem, R. A. K. Sherwani, and C. H. Jun, "Monitoring mortality caused by COVID-19 using gamma-distributed variables based on generalized multiple dependent state sampling," Computational and Mathematical Methods in Medicine, vol. 2021, Article ID 6634887, 17 pages, 2021. 